\title{
EDITORIAL
}

\section{Thank You to Reviewers}

\author{
Thomas M. Biggs
}

Published online: 21 January 2010

(c) Springer Science+Business Media, LLC and International Society of Aesthetic Plastic Surgery 2010

Dear Readers:

Last year I wrote the following words regarding the faceless and nameless (in this circumstance) people who make up the "Peers" of the peer reviewed Journal. I can't make the words say my feelings any better.

It is with great appreciation that the Journal publishes the list of Reviewers for 2009. The number of cogs in the wheel of journal publishing are numerous, but none for a peer-reviewed journal more than the peers themselves. We are happy to express our gratitude for their excellent and untiring work. To the public they are unrecognized, but to the Editorial Board they are the Journal's foundation.

Elie Abdelhak

Tawfeik Alyafi

Ana Badin

Ricardo Baroudi

Alfonso Barrera

Hilton Becker

Alexei Borovikov

James Boynton

Mitchell Brown

Louis Bucky

Andre Camirand

Yi Lin Cao

Lloyd Carlsen

Jenny Carvajal

Linda Cendales

Nazim Cerkes

Abel Chajchir

Yu-Ray Chen

T. M. Biggs $(\bowtie)$

1315 St. Joseph Parkway, Suite 900, Houston, TX 77002, USA

e-mail: tbiggsmd@aol.com

\author{
Ernest Chiu \\ Mark Codner \\ Sydney R. Coleman \\ Miodrag M. Colic \\ Gustavo Colon \\ Ernest Cronin \\ Bruce Cunningham \\ Jorge De La Torre \\ Ewaldo Bolivar De Souza Pinto \\ Roy De Vita \\ Jose Luis M. Del Yerro \\ Marita Eisenmann-Klein \\ Hisham Mahmoud El Minawi \\ Franklyn Elliott \\ John Eng \\ Onur Erol \\ Joel Feldman \\ Marcus Ferreira \\ Julius Few \\ Peter Bela Fodor \\ Alain L. Fogli \\ Antonio Fuente Del Campo \\ Julio Garcia \\ Rolf Gemperli \\ Alaa Gheita \\ Joao Carlos Sampaio Goes \\ Ruth Graf \\ James Grotting \\ Ronald P. Gruber \\ Wolfgang Gubisch \\ Fernando Guerrerosantos \\ Jose Guerrerosantos \\ Ashok Gupta \\ Dennis Hammond \\ Sam Hamra
}


Per Hedén

Jorge Alberto Herrera

Darryl James Hodgkinson

Joseph Hunstad

Spyro V. Joannides

Michael A.C. Kane

Nolan S. Karp

Umar D. Khan

Kamran Khoobehi

Brian Kinney

Stanely Klatsky

David Knize

Paraskevas Kontoes

Lawrence Koplin

Val Lambros

Claude Le Louarn

John William Little

Federico Mayo

Bryan C. Mendelson

Henry Mentz

Nikolaos Metaxotos

Sebastian Mir-Mir Caragol

Paolo G. Morselli

Antonio Aldo Mottura

Egle Muti

Farzad R. Nahai

Foad Nahai

Fabio Xerfan Nahas

James D. Namnoum

Constance Neuhann-Lorenz

German Newall

Donald Nuss

Kitaro Ohmori

Daehwan Park

Fereydoun Don Parsa

Jean Francois Pascal
Neeta Patel

Malcolm Paul

Carlton Perry

Jan Gordon Poell

Arturo Prado

Kirill Pshenisnov

Lee L.Q. Pu

Oscar Ramirez

Gino Rigotti

Peter Rubin

Adam Rubinstein

Richard Sadove

Jaime O. Salcedo-Martinez

Osvaldo Saldanha

Renato Saltz

Jun-Ichi Sanuki

Michael Scheflan

Mark A. Schusterman

Gaith Shubailat

Ozan Sozer

Scott L. Spear

Melvin Spira

Ithamar Nogueira Stocchero

Susumu Takayanagi

Patrick L. Tonnard

Bryant Alexander Toth

Carlos Oscar Uebel

Edwin Vasquez

Alexis M. Verpaele

Jennifer Walden

Russell John Walton

Thomas C. Wiener

Roger Wixtrom

Woffles Wu

Song-Lin Yang

Michael J. Yaremchuk 